\title{
IL-6 Overexpression in ERG-Positive Prostate Cancer Is Mediated by Prostaglandin Receptor EP2
}

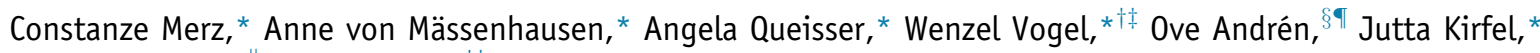
Stefan Duensing, "Sven Perner, ${ }^{* \dagger}$ and Michael Nowak*

From the Section of Prostate Cancer Research,* Institute of Pathology, Center for Integrated Oncology Cologne/Bonn, University Hospital of Bonn, Bonn, Germany; the Pathology Department, ${ }^{\dagger}$ University Hospital of Luebeck, Luebeck, Germany, the Leibniz Research Center Borstel, ${ }^{\ddagger}$ Borstel, Germany; the Department of Urology, ${ }^{\S}$ University Hospital of Örebro, Sweden; the Institute of Health and Medical Science, ${ }^{\uparrow}$ University of Örebro, Sweden; and the Section of Molecular Urooncology," Department of Urology, University of Heidelberg School of Medicine, Heidelberg, Germany

Accepted for publication December 8, 2015.

Address correspondence to Michael Nowak, Ph.D., Institute of Pathology, University Hospital of Bonn, SigmundFreud-Strasse 25, 53127 Bonn, Germany. E-mail: nowakm@ uni-bonn.de.

\begin{abstract}
Prostate cancer is the most diagnosed cancer in men and multiple risk factors and genetic alterations have been described. The TMPRSS2-ERG fusion event and the overexpression of the transcription factor ERG are present in approximately $50 \%$ of all prostate cancer patients, however, the clinical outcome is still controversial. Prostate tumors produce various soluble factors, including the pleiotropic cytokine IL-6, regulating cellular processes such as proliferation and metastatic segregation. Here, we used prostatectomy samples in a tissue microarray format and analyzed the co-expression and the clinicopathologic data of ERG and IL-6 using immunohistochemical double staining and correlated the read-out with clinicopathologic data. Expression of ERG and IL- 6 correlated strongly in prostate tissue samples. Forced expression of ERG in prostate tumor cell lines resulted in significantly increased secretion of IL- 6 , whereas the down-regulation of ERG decreased IL-6 secretion. By dissecting the underlying mechanism in prostate tumor cell lines we show the ERG-mediated up-regulation of the prostanoid receptors EP2 and EP3. The prostanoid receptor EP2 was overexpressed in human prostate cancer tissue. Furthermore, the proliferation rate and IL- 6 secretion in DU145 cells was reduced after treatment with EP2-receptor antagonist. Collectively, our study shows that the expression of ERG in prostate cancer is linked to the expression of IL-6 mediated by the prostanoid receptor EP2. (Am J Pathol 2016, 186: 974-984; http://dx.doi.org/ 10.1016/j.ajpath.2015.12.009)
\end{abstract}

Prostate cancer ( $\mathrm{PCa}$ ) is the most diagnosed cancer in men worldwide and remains a leading cause for cancer-related death. The prevalence of PCa is increasing in the Western world as a result of the elderly population and improved screening methods.

Overexpression of the erythroblast transformationspecific family transcription factor erythroblast transformation-specific-related gene $(E R G)$ is a common event in tumor development. Erythroblast transformation-specific factors are expressed in various cell types and regulate a plethora of cellular functions, such as angiogenesis, growth, differentiation, and survival. ${ }^{1}$ One of the most frequent genomic aberrations in prostate cancer is the fusion of ERG with the promoter region of transmembrane protease, serine 2 (TMPRSS2). Depending on the study design, the frequency rate of $T M P R S S 2-E R G$ fusion events range from
$15.3 \%$ to $79.3 \% .^{2}$ TMPRSS2 is an androgen-regulated gene, hence, fusion of $E R G$ to the promoter region of TMPRSS2 results in androgen-induced overexpression of ERG. ${ }^{3}$ However, the clinical impact of TMPRSS2-ERG

Supported by grants from the Rudolf Becker-Foundation, grant 2011.077.2 from the Wilhelm Sander Foundation (S.P.), grants DFG PE1179/9-1 and PE1179/11-1 (S.P.) and DFG NO787/5-1 (M.N.) from the German Research Foundation, and a medical doctoral fellowship grant BONFOR O-155.0064 from the University Hospital of Bonn (C.M.).

S.P. and M.N. contributed equally to this work as senior authors.

Disclosures: The Brigham and Women's Hospital and the University of Michigan have filed a patent on erythroblast transformation-specific gene rearrangements in prostate cancer, on which S.P. is a co-inventor and the diagnostic field of use has been licensed to GenProbe, Inc. GenProbe has neither played a role in the design and conduct of the study, nor in the collection, analysis, or interpretation of the data, and has no involvement in the preparation, review, or approval of the manuscript. 
fusion and subsequently the overexpression of ERG remain controversial. ${ }^{4}$ Early studies have described an association of $E R G$ rearrangement with greater invasion, $\mathrm{PCa}$-specific death, and ERG as a prognostic factor for disease recurrence. In contrast, recent studies have shown ERG expression is indicative of lower pathologic grade, lower Gleason score, longer recurrence-free survival, or have described no correlation with outcome. ${ }^{2}$ These divergent results seem to depend on the detection techniques used (fluorescent in situ hybridization, RT-PCR, or immunohistochemistry), sample acquisition technique, and sample size. Taken together, TMPRSS2-ERG fusion and ERG overexpression represent a molecular subtype of $\mathrm{PCa}$, but the functional consequences and interplay with other tumor-derived factors remain to be clarified.

In addition to chromosomal alterations, various growth factors and cytokines impact tumor development and progression. Of such cytokines, IL-6 is a frequent product of tumors. ${ }^{5}$ IL-6 affects growth, differentiation, and apoptosis in various cancers, including those of the prostate. ${ }^{6}$ Effects of IL- 6 are mediated by the IL- 6 -receptor complex (glycoprotein 80). Upon IL-6 binding, gp130 dimerizes and initiates intracellular signaling through Janus kinase activation, which is followed by phosphorylation, nuclear translocation, and DNA binding of STAT3. ${ }^{7}$ Patients with PCa show increased serum levels of IL- 6 and the probability of a biochemical recurrence increases with higher serum levels of IL- $6 .{ }^{8}$ Furthermore, PCa progression is accelerated after long-term IL-6 exposure in vitro, supporting the notion that IL-6 is a potential biomarker for PCa development and progression. However, the mechanisms governing IL-6 expression in $\mathrm{PCa}$ are poorly understood. Both IL-6 as part of chronic inflammation and IL-6 expressed through autocrine and paracrine activation via STAT3 phosphorylation have been described. ${ }^{9}$

Another important key product of tumors, including those of the prostate, is prostaglandin $\mathrm{E}_{2}$ (Pge2). Pge2 is a lipid compound and end product of eicosanoid synthesis, which involves the enzymes cyclooxygenase (COX)-1 and COX-2. COX inhibitors such as indomethacin and celecoxib have been shown to have antineoplastic effects. ${ }^{10}$ Pge2 binds to a family of four receptors, termed prostanoid receptors, EP1 to EP4. EP1 to EP4 are encoded by the genes PTGERI to PTGER4 and share significant sequence homology. Despite these similarities, prostanoid receptors are linked to a diverse set of intracellular signaling events. ${ }^{11} \mathrm{EP} 1$ is $\mathrm{G}_{\mathrm{q}}$-coupled and, hence, binding of this receptor increases intracellular $\mathrm{Ca}^{2+}$ levels and results in activation of phospholipase A2. ${ }^{12}$ EP2 and EP4 are $\mathrm{G}_{\mathrm{s}}$-coupled proteins and activate adenylyl cyclase, followed by activation of protein kinase $A$. In contrast, EP3 is coupled to $\mathrm{G}_{\alpha \mathrm{i}}$ protein and binding decreases the formation of cAMP. ${ }^{13}$ Expression of prostanoid receptors is deregulated in many human tumor entities. ${ }^{14}$ In prostate cancer cell lines, deregulation and effects of different prostanoid receptors are discussed controversially. Vo et $\mathrm{al}^{15}$ showed that Pge2 mediates its effects on migration mediated by EP4. In contrast, Kashiwagi et al ${ }^{16}$ showed that EP3 contributes to castration resistance in prostate cancer. To date, only Huang et al, ${ }^{17}$ using a small cohort size, have reported an up-regulation of both EP2 and EP4 in PCa and independent effects of EP3 and EP4 on in vitro migration of the PCa cell line PC-3.

Here, we evaluated if the transcription factor ERG controls the expression of the cytokine IL-6 in PCa. We show that the expression of ERG in human prostate cancer significantly correlates with IL-6 expression and EP2 expression in prostate tissues. We further provide evidence that ERG controls IL-6 production in PCa cell lines by effects mediated mainly by the prostanoid receptor EP2.

\section{Material and Methods}

\section{Ethical Standards}

This study was approved by the Institutional Review Board of the University of Uppsala/Örebro, Sweden (2009/016) and the Institutional Review Board of the University Hospital of Bonn, Germany (2013/010), and all patients provided their informed consent before their inclusion in the study. The study therefore is in accordance with the ethical standards laid down in the 1964 Declaration of Helsinki and its later amendments.

\section{Cell Lines and Reagents}

Immortalized PCa cell lines (DU145, PC-3, and VCaP) originally were obtained from the ATCC and cultured according to the manufacturer's instructions. DU145 and PC-3 cells were grown in RPMI-1640 medium (Merck Millipore, Darmstadt, Germany) supplemented with $10 \%$ fetal calf serum, $1 \%$ penicillin/streptomycin, and 1\% L-glutamine. VCaP cells were grown in Dulbecco's modified Eagle's medium (Life Technologies, Darmstadt, Germany) supplemented with $10 \%$ fetal calf serum and $1 \%$ penicillin/streptomycin. All cell lines were verified recently by Multiplexion (Heidelberg, Germany). Unless otherwise stated, chemicals were obtained from SigmaAldrich (Taufkirchen, Germany).

\section{Patient Cohort}

PCa tissues were obtained during radical prostatectomies performed at the University Hospital of Örebro, Sweden, between 1989 and 2005, and immediately fixed in 20\% formalin. For tissue microarray construction three representative $0.6-\mathrm{mm}$ cores from the circled cancer tissue specimens as well as one benign focus were selected randomly and were reviewed by the study pathologist (S.P.). Clinicopathologic data of the patient cohort were previously described by Nowak et al, ${ }^{18}$ and are summarized in Table 1 .

\section{Immunohistochemistry}

Immunohistochemical detection of different proteins was performed using the Ventana Discovery XT automated 
Table 1 Clinicopathologic Characteristics of Patients Included in the Study

\begin{tabular}{|c|c|c|}
\hline \multicolumn{3}{|l|}{ Patient } \\
\hline Parameters & Total & $\%$ \\
\hline $\mathrm{N}$ & 311 & 100 \\
\hline Malignant tissue & 218 & 70.1 \\
\hline Benign tissue & 93 & 29.9 \\
\hline Age, median (range), year & \multicolumn{2}{|l|}{$63(45-74)$} \\
\hline Follow-up time, median (range), months & \multicolumn{2}{|l|}{$122(46-273)$} \\
\hline $\begin{array}{l}\text { PSA at diagnosis, median (range), } \mathrm{ng} / \mathrm{mL} \\
\text { Gleason group }\end{array}$ & \multicolumn{2}{|l|}{$8.8(3-52)$} \\
\hline$\leq 6$ & 51 & 23.4 \\
\hline $3+4$ & 57 & 26.1 \\
\hline $4+3$ & 82 & 37.6 \\
\hline$\geq 8$ & 28 & 12.9 \\
\hline \multicolumn{3}{|l|}{ pT stage } \\
\hline $\mathrm{T} 1 \mathrm{a}$ & 1 & 0.5 \\
\hline $\mathrm{T} 1 \mathrm{~b}$ & 6 & 2.7 \\
\hline T1c & 112 & 51.4 \\
\hline $\mathrm{T} 2$ & 76 & 34.9 \\
\hline T3 & 9 & 4.1 \\
\hline Not available & 14 & 6.4 \\
\hline \multicolumn{3}{|l|}{ CRPC } \\
\hline Yes & 16 & 7.3 \\
\hline No & 187 & 85.8 \\
\hline Not available & 15 & 6.8 \\
\hline \multicolumn{3}{|l|}{ PSA relapse } \\
\hline Yes & 59 & 27.1 \\
\hline No & 132 & 60.6 \\
\hline Not available & 27 & 12.3 \\
\hline \multicolumn{3}{|l|}{ Overall death } \\
\hline Yes & 47 & 21.6 \\
\hline No & 157 & 72.0 \\
\hline Not available & 14 & 6.4 \\
\hline \multicolumn{3}{|l|}{ PCa-specific death } \\
\hline Yes & 13 & 6.0 \\
\hline No & 191 & 87.6 \\
\hline Not available & 14 & 6.4 \\
\hline
\end{tabular}

PSA, prostate-specific antigen; $\mathrm{pT}$, pathological tumor stage.

staining system (Ventana Medical Systems, Tucson, AZ). Paraffin-embedded tissue microarray blocks were sectioned into 4- $\mu \mathrm{m}$ slices, deparaffinized, and antigens were demasked in EDTA buffer ( $\mathrm{pH}$ 8.4). Primary antibodies used were specific for ERG (clone EPR3864, ready to use; Ventana Medical Systems, Tucson, AZ), EP1 to EP4 (all polyclonal rabbit Ig; all Cayman Chemical, Ann Arbor, MI), or IL-6 (polyclonal rabbit Ig; Abcam, Cambridge, MA). Secondary staining was conducted using a biotin-free detection kit (UltraMap anti-Rb Detection Kit). Color was developed using alkaline phosphatase (UltraMap anti-Rb Alk Phos; Ventana Medical Systems) for IL-6 or 3-3'-diaminobenzidine (UltraView Universal Diaminobenzidine Detection kit; Ventana Medical Systems) for all other antigens. Finally, slides were counterstained with Hematoxylin II and Bluing Reagent (Ventana Medical Systems).

\section{Expression Analysis}

ERG and IL-6 protein expression were assessed and quantified manually as well as digitally. The manual expression analysis was performed by a pathologist (S.P.). ERG protein expression was distinguished into positive and negative, and the IL-6 expression was graduated according to the Remmele score into four categories: negative, low, intermediate, and high positive. For the digital expression analysis slides were digitized (Pannoramic Desk; 3DHistech, Budapest, Hungary). Semiautomated discrimination of tumor areas and expression analysis of ERG, IL-6, and EP1 to EP4 was performed using Definiens Tissue Studio 2.1 image analysis software (Definiens, Munich, Germany).

\section{Transfection}

For overexpression of ERG, $1 \mu \mathrm{g}$ DNA of the pBabe-green fluorescent protein vector inserted with ERG or the empty control pBabe vector was used and transfected using ScreenFect A (Genaxxon Bio Science, Ulm, Germany) following the manufacturer's instructions. A total of 5000 cells were plated in antibiotic-free medium in a 96-well plate. After 24 hours the medium was changed. Supernatants were taken after a further 24 hours and IL-6 levels were measured by enzyme-linked immunosorbent assay. Clones stably transfected with ERG-pBabe vector or empty vector were selected by repeated flow-sorting green of fluorescent protein-positive cells (FACSAriaIII; BD Biosciences, Heidelberg, Germany).

Transient knockdown of ERG or PTGER2 was performed by Screenfect A-mediated lipofection (Incella, EggensteinLeopoldshafen, Germany), with 50 pmol target-specific siRNA (SMARTpool siGENOME) or scrambled siRNA (both Thermo Fisher Scientific, Waltham, MA) according to the manufacturer's instructions.

\section{Enzyme-Linked Immunosorbent Assay}

Levels of IL-6 in cell culture supernatants were determined by standard sandwich enzyme-linked immunosorbent assay using matched antibody pairs (eBioscience, San Diego, CA). Color reactions were developed by incubation with 3,3',5,5'-tetramethylbenzidine substrate (Biolegend, San Diego, CA), and stopped using $0.5 \mathrm{~mol} / \mathrm{L} \mathrm{H}_{3} \mathrm{PO}_{4}$. OD was read with a microplate reader (Epoch; BioTek Instruments, Bad Friedrichshall, Germany) at $450 \mathrm{~nm}$. Pge2 levels in cell culture supernatants were determined by an enzyme-linked immunosorbent assay kit (Life Technologies, Darmstadt, Germany) according to the manufacturer's instructions.

\section{Western Blot Analysis}

Cultured cells were harvested and lysed in RIPA buffer (150 $\mathrm{mmol} / \mathrm{L} \mathrm{NaCl}, \quad 1.0 \%$ IGEPAL NP-40, $0.5 \%$ sodium deoxycholate, $0.1 \%$ SDS, $50 \mathrm{mmol} / \mathrm{L}$ Tris, $\mathrm{pH} 8.0$ ) for 1 
hour and centrifuged at $30,000 \times g$ for 30 minutes. Protein concentration was determined using the Bio-Rad (Munich, Germany) BCA assay. Proteins were transferred to a polyvinylidene difluoride membrane, blocked in 5\% milk, and incubated overnight with their respective primary antibodies diluted in Tris-buffered saline $/ 0.05 \%$ Tween-20/5\% milk. After extensive washing with Tris-buffered saline/ $0.05 \%$ Tween-20, the membranes were incubated with horseradish peroxidase-conjugated antibodies against rabbit or mouse Ig, and developed using ECL Western Blotting Reagent (GE Healthcare, Frankfurt, Germany).

\section{Real-Time PCR}

RNA was extracted using the RNeasy Mini Kit (Qiagen, Hilden, Germany) and mRNA was transcribed into cDNA (iScript cDNA Synthesis Kit; Bio-Rad) according to the manufacturer's instructions.

mRNA expression of various genes was measured by realtime PCR (LightCycler 480; Roche, Basel, Switzerland) using the following primer sequences: ERG, 5'-GACGACTTCCAGAGGCTCAC- $3^{\prime}$ (forward) and 5'-ATAAAAGCTGCACCCCCTGT- ${ }^{\prime}$ (reverse); $\beta$-actin $5^{\prime}$-AGTCCTGTGGCATCCACGAAACT- $3^{\prime}$ (forward) and $5^{\prime}$-CACTGTGTTGGCGTACAGGTCTT-3' (reverse); PTGER1 (EP1), $5^{\prime}$-ATCATGGTGGTGTCGTGCAT-3' (forward) and $5^{\prime}$ TACACCCAAGGGTCCAGGAT- ${ }^{\prime}$ (reverse); PTGER2 (EP2), 5'-CTCGCCTGCAACTTCAGTGT-3' (forward) and $5^{\prime}$-AGGCCTAAGGATGGCAAAGAC-3' (reverse); PTGER3 (EP3), 5'-GACACACACGGAGAAGCAGA-3' (forward) and 5'-CACAATGTGCAGTTGCCCTC-3' (reverse); PTGER4 (EP4), 5'-CTGCTCCATCCCGCTCG-3' (forward) and $5^{\prime}$ CTGTCTGAGCAGTGCTGTCC-3' (reverse); PLAU (uPa), 5'-GGTCAATCCATTTCTCCTGGCT-3' (forward) and 5'CTTTAGGCCCACCTGCACATA-3' (reverse); hydroxyprostaglandin dehydrogenase-15(NAD) (HPGD), 5'-AACCTCAGAAGACTCTGTTCATCC- $3^{\prime}$ (forward) and $5^{\prime}$ CCAAAATGTCCAGTCTTCCAAAGT- $3^{\prime}$ (reverse); IL-1 $\beta$, $5^{\prime}$-CAACAGGCTGCTCTGGGATT- $3^{\prime}$ (forward) and $5^{\prime}$ CATGGCCACAACAACTGACG-3' (reverse). Expression levels were calibrated to levels of $\beta$-actin as housekeeping gene in the same sample.

\section{In Silico Promoter Analysis}

Promoter regions of indicated genes ranging from the transcription start site $(0 \mathrm{~kb})$ to $-7 \mathrm{~kb}$ upstream were screened for ERG-binding sites (EBS) (Jaspar database ID MA0474.2) using the JASPAR core database (Find Individual Motif Occurrences, http://jaspar.genereg.net, last accessed November 17, 2015). ${ }^{19}$

\section{Statistics}

Statistical analyses were performed using GraphPad Prism V (GraphPad Software, San Diego, CA). Statistical significance was calculated using the $t$-test or one-way analysis of variance with the Tukey post-test, and correlations were calculated using the Pearson correlation coefficient. $P<0.05$ was considered statistically significant.

\section{Results}

\section{ERG Protein Expression Strongly Correlates with IL-6 Expression}

Tissue microarrays consisting of benign and tumor tissues were immunostained against ERG and IL-6. All four combinations of ERG and IL-6 staining could be distinguished (Figure 1, A-D). ERG immunostaining was located in the nucleus of prostate cells and was dichotomized into positive or negative immmunostaining. Thirty-nine percent of all cancer cells showed a positive expression of ERG whereas none of the benign tissue samples had a positive ERG expression, underlining the fact that ERG is specific for malignant prostate tissue (Table 2). IL-6 immunostaining was located in the cytoplasm of prostate cells and staining intensity was determined. Fourteen percent of the tumor samples were considered negative or weakly IL-6-positive, $48 \%$ were classified as intermediate-positive, and $34 \%$ of the tumor showed strong IL-6 staining. In contrast, the benign tissue showed a lower IL-6 intensity: $28 \%$ of the samples were considered low positive, $60 \%$ as intermediate, and only $7 \%$ were strong positive for IL-6 (Table 2). Having assessed the expression of ERG and IL-6 separately, we analyzed the co-expression of these two parameters in all foci. The IL-6 intensity was significantly higher in ERG-positive foci than in ERG-negative foci (Figure 1E). We further confirmed this correlation by digital quantification. Here, all tumor samples were stratified according to quartiles of ERG expression (Figure 1F). IL-6 expression in samples of the first quartile of ERG expression was significantly lower than in the other quartiles; moreover, samples in the second quartile showed lower IL-6 expression than ERG-high-expressing tumors (Figure 1F).

\section{ERG Controls the Secretion of IL- 6 in Prostate Cancer Cell Lines}

The observed co-expression of ERG and IL-6 in prostate cancer tissue prompted us to test if ERG controls the expression of IL-6. To this end, DU145 cells were transfected with an ERG-encoding expression vector. Clones stably overexpressing ERG or containing the empty control vector were selected by repeated flow-sorting green fluorescent protein-positive cells. Forced expression of ERG in these cells led to a significant increase in ERG mRNA and protein expression (Figure 2, A and B). ERG-overexpressing DU145 cells showed significantly higher IL-6 production compared 
A
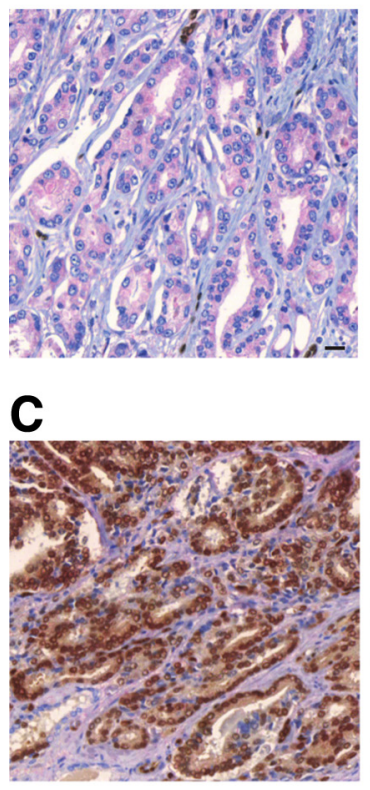

$\mathbf{E}$

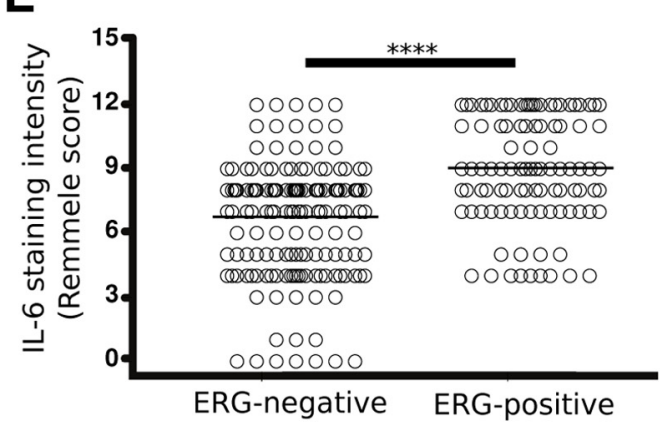

B
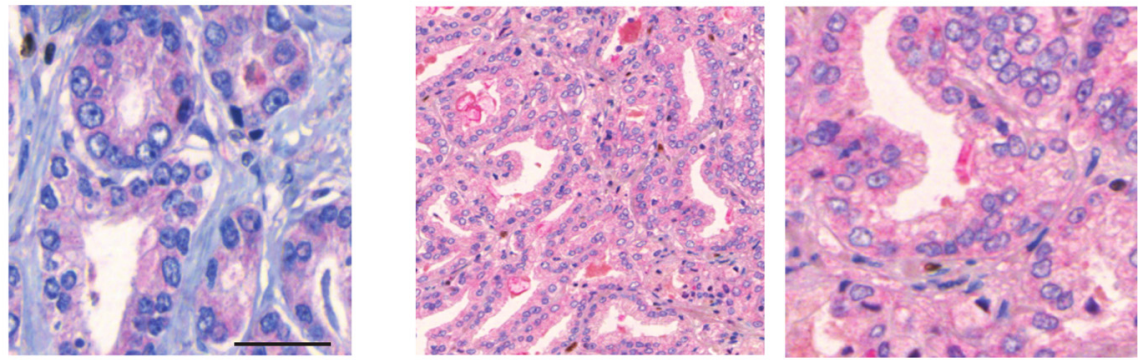

D
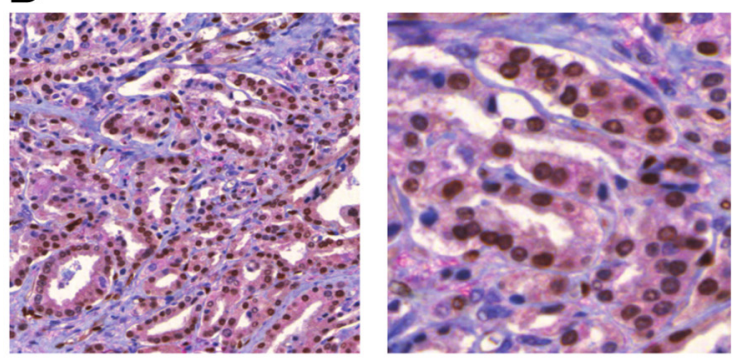

$\mathbf{F}$

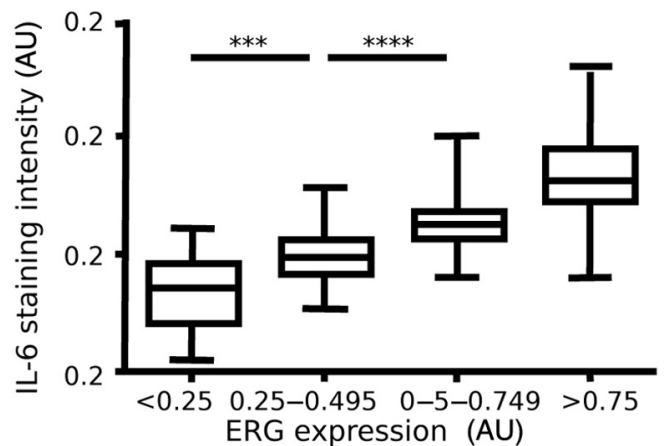

Figure 1 A-D: IL-6 in prostate tumors co-expresses with ERG. Representative immunohistochemical images of ERG-negative/IL-6 low-positive (A), ERGnegative/IL-6 high-positive (B), ERG-positive/IL-6 low-positive (C), and ERG-positive/IL-6 high-positive (D) staining. Cytoplasmic red stain represents IL-6 and brown nuclear staining represents ERG. E: Mean IL-6 intensity in ERG-positive or -negative cores according to the Remmele score. F: IL-6 intensity and ERG expression in all cores as determined by semiautomated quantification. Cores were stratified into quartiles of ERG expression. ${ }^{* *} P<0.005,{ }^{* * * *} P<0.001$. Scale bars: $20 \mu \mathrm{m}$. AU, arbitrary unit; ERG, erythroblast transformation-specific-related gene.

with control cells (Figure 2C). To further show that ERG expression drives IL-6 production we used $\mathrm{VCaP}$ harboring the TMPRSS2-ERG translocation. siRNA-mediated knockdown of ERG in these cells led to a robust abrogation of ERG transcripts and ERG protein, whereas transfection with scrambled siRNA did not reduce ERG (Figure 2D). In VCaP cells harboring the TMPRSS2-ERG fusion, knockdown of

Table 2 Expression of ERG and IL- 6 in Benign and Malignant Prostate Biopsy Specimens

\begin{tabular}{lcr}
\hline Foci $(n=398)$ & Malignant & \multicolumn{1}{l}{ Benign } \\
\hline ERG positive $(n=119)$ & $39 \%(n=119)$ & $0 \%(n=0)$ \\
ERG negative $(n=279)$ & $61 \%(n=184)$ & $100 \%(n=95)$ \\
IL-6 negative & $3.3 \%(n=10)$ & $4.2 \%(n=4)$ \\
IL-6 low $(0-3)$ & $14.2 \%(n=43)$ & $28.4 \%(n=27)$ \\
IL-6 intermediate $(4-8)$ & $48.2 \%(n=146)$ & $60 \%(n=57)$ \\
IL-6 high (9-12) & $34.3 \%(n=104)$ & $7.4 \%(n=8)$ \\
\hline
\end{tabular}

ERG significantly reduced the expression of IL-6 (mean reduction, 27-fold) (Figure 2E).

\section{ERG Overexpression Is Correlated to Prostaglandin Receptors}

To further address the mechanisms mediating IL-6 upregulation, we subjected cells stably transfected with ERG to expression analysis. Given the unresolved link between prostaglandin-receptor signaling and IL-6 expression, we focused on genes that could be functionally linked to prostaglandin signaling. DU145 cells expressing ERG expectedly showed a strong up-regulation of the transcription factor ERG in comparison with control cells on mRNA level and protein (Figure 3, A and B). Furthermore, corroborating the work of Mohamed et al, ${ }^{20}$ forced expression of ERG led to a marked down-regulation of the prostaglandin-catabolizing enzyme HPGD (Figure 3A). 

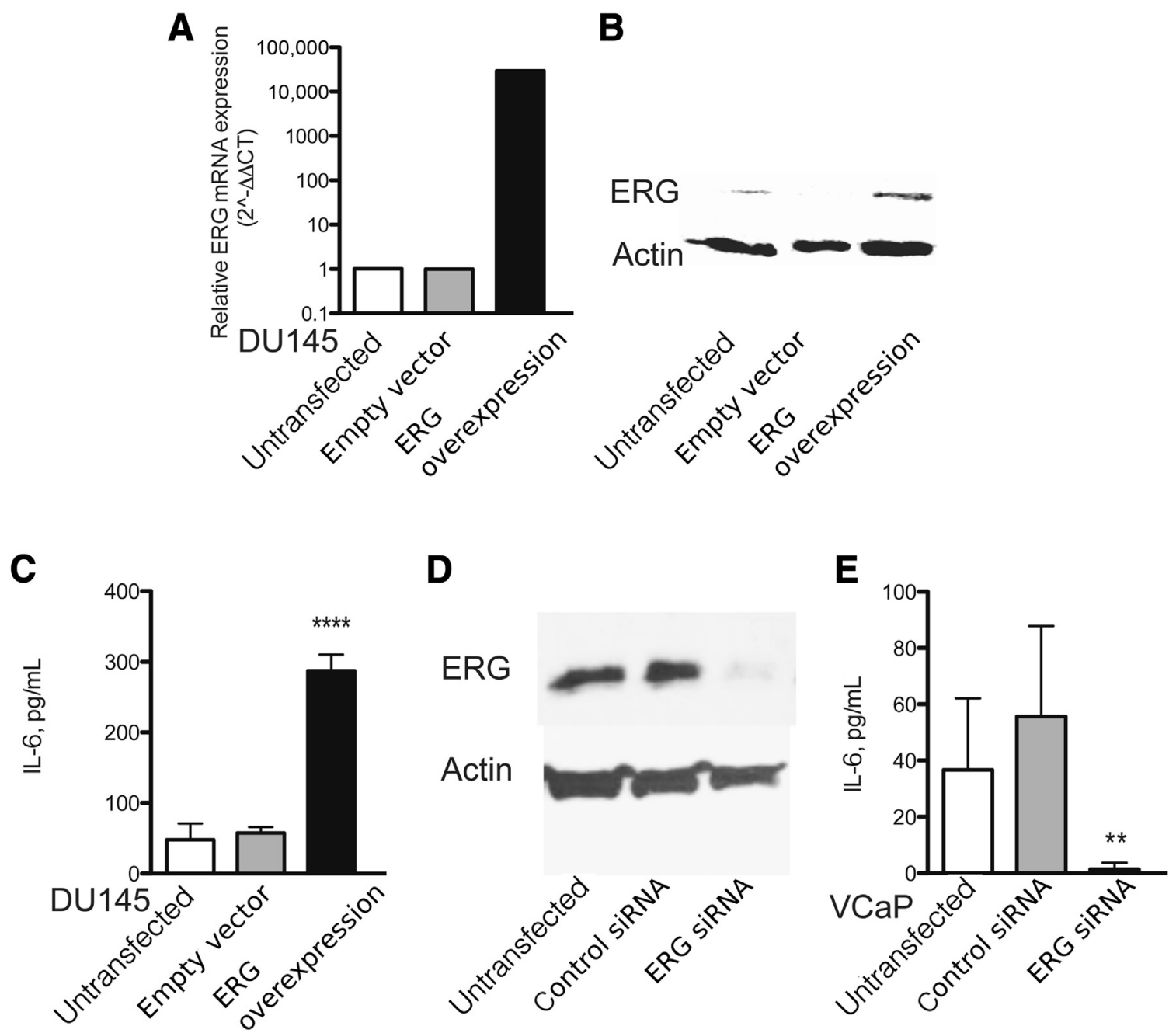

Figure 2 ERG controls the production of IL- 6 in prostate tumor cell lines. A and B: DU145 cells were transfected with an ERG expression vector, empty vector, or left untreated. ERG overexpression was measured by real-time PCR (A) and Western blot analysis (B). C: IL-6 content in cell culture supernatants was analyzed by enzyme-linked immunosorbent assay. D: ERG protein expression 48 hours after transfection with ERG-specific or scrambled siRNA in VCaP cells. E: Knockdown of ERG in VCaP cells abolishes IL-6 production, as measured by enzyme-linked immunosorbent assay. Results are presented as means \pm SD, representative of at least three experiments. ${ }^{* *} P<0.01,{ }^{* * * *} P<0.001$. ERG, erythroblast transformation-specific - related gene.

As an essential enzyme of prostaglandin metabolism, HPGD catalyzes the oxidation of prostaglandins into inactive metabolites. The down-regulation of HPGD in ERG-overexpressing cells prompted us to evaluate different elements of prostaglandin metabolism. To this end we also analyzed the mRNA expression level of prostaglandin-receptor genes PTGERl to 4 in DU145 cells. In response to overexpression of ERG, the mRNA expression of the prostaglandin receptor EP4 (PTGER4) increased by 2.8 -fold, whereas transcription of genes encoding the other prostanoid receptors was less than twofold. We further tested the mRNA expression of $P L A U$, a gene encoding for the urokinase-type plasminogen activator, which is up-regulated by prostaglandins and thus aggravates prostate tumor growth. ${ }^{21}$ However, and in contrast to the aforementioned IL-1 $\beta$, PLAU expression was not regulated more than twofold compared with control cells (Figure 3A).
After we examined the gene expression level of the prostanoid receptors (PTGERI through 4), we evaluated the protein expression of these receptors. The protein expression of EP2 and EP3 strongly increased in ERGoverexpressing DU145 cells. In contrast, the protein expression of EP4 did not increase (Figure 3B). The results do not indicate one specific prostanoid receptor in ERGexpressing cells, therefore further regulatory mechanisms have to be verified.

DU145 cells stably transfected with ERG strongly upregulated the transcription of the cytokine IL-1 $\beta$ (Figure 3A). Notably, in addition to its multiple functions as an inflammatory mediator, IL-1 $\beta$ is a potent inducer of COX, which catalyzes the formation of prostaglandins. ${ }^{22}$ In DU145 cells transfected with ERG, we detected a significantly higher release of IL-1 $\beta$ (Figure 3C). Forced expression of ERG in DU145 directly up-regulated the production of Pge2, compared with untransfected DU145 cells (Figure 3D). 


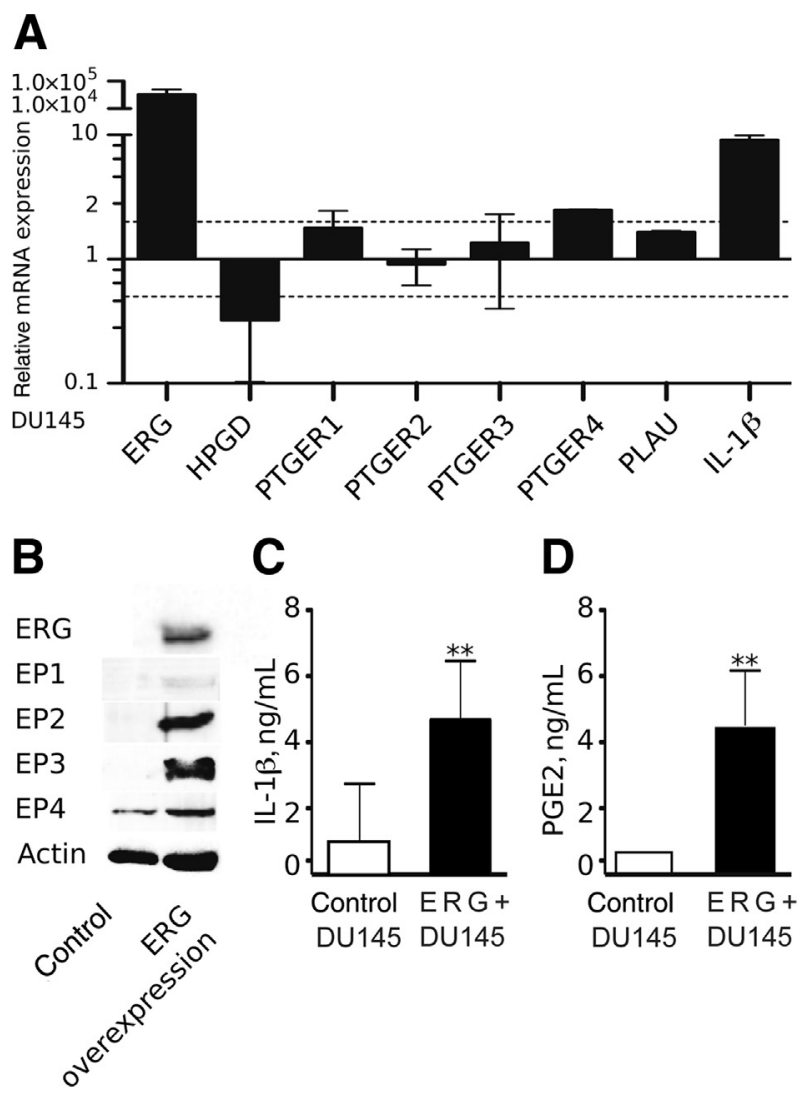

E
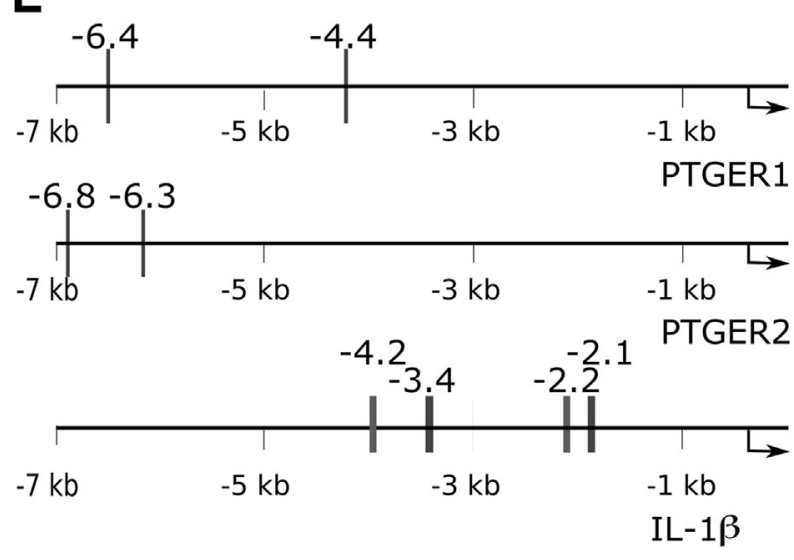

Figure 3 Forced overexpression of ERG in DU145 cells results in upregulation of prostanoid receptors EP2 and EP3. A: mRNA expression profile of prostaglandin receptors (EP1 through EP4), prostaglandin catabolism (HPGD), urokinase ( $\mathrm{UPa})$, and IL-1 $\beta$ in prostate tumors stably transfected with ERG relative to levels in empty vector-transfected cells, assessed by real-time PCR. B: Lysates of DU145 cells stably transfected with ERG or empty vector were subjected to Western blot analysis of the indicated proteins, and $\beta$-actin was used as loading control. C: IL-1 $\beta$ release into cell culture medium of ERGtransfected or control plasmid-transfected DU145 cells, as measured by enzyme-linked immunosorbent assay. D: Pge2 release into cell culture medium of ERG-transfected or control plasmid-transfected DU145 cells, as measured by enzyme-linked immunosorbent assay. Results are representative of three experiments yielding comparable results. E: ERG-binding sites in promoter regions ( $-7 \mathrm{~kb}$ to transcription start) of prostanoid receptors PTGER1, PTGER2, and IL-1 $\beta$ identified in silico using the FIMO (Find Individual Motif Occurrences) suite. In promoter regions of prostanoid receptors PTGER3 and PTGER4 no ERG-binding sites are identified. $n=3(\mathbf{A}-\mathbf{D}) .{ }^{*} P<0.01$. ERG, erythroblast transformation-specific-related gene.

The complex regulation of prostanoid-receptor transcripts by the transcription factor ERG prompted us to screen the promoter regions of the genes of these receptors as well as IL-1 $\beta$ for occurrence of EBS. By using the publicly available JASPAR database we screened the DNA upstream to a distance of $7 \mathrm{~kb}$ of the respective transcription start site for screening against the ERGbinding motif (ID MA0474.2). ${ }^{23}$ By using this approach we identified two EBS at $-4.4 \mathrm{~kb}$ and $-6.4 \mathrm{~kb}$ in the promoter region of PTGER1, as well as two EBS at -6.3 $\mathrm{kb}$ and $6.8 \mathrm{~kb}$ in the $P T G E R 2$ promoter, using a threshold for each of $90 \%$. In contrast, no EBS could be identified in the promoter regions of PTGER3 or PTGER4. Moreover, the promoter of IL- $1 \beta$, which among the tested genes showed the strongest mRNA regulation by ERG (Figure 3A), harbors four EBS within a distance of $4.2 \mathrm{~kb}$ from the transcription start site (Figure 3E).

\section{Prostaglandin Receptors Mediate Proliferation and IL-6 Production of ERG-Expressing Cells}

Having shown that prostanoid receptors EP2, EP3, and EP4 are expressed in DU145 cells transfected with ERG, we further determined which of these three receptors controls the proliferation of tumor cells. We cultured DU145 cells transfected with ERG expression vector or the control construct for 48 hours in the presence of prostanoid-receptor-specific antagonists and tested the cell-proliferative capacity. ERGexpressing DU145 cells showed significantly increased proliferation compared with control DU145 cells (Figure 4, A-C). DU145-ERG cells incubated with concentrations of 25 and $50 \mathrm{nmol} / \mathrm{L}$ of the EP2-specific antagonist PF04418948 showed no more significant difference in proliferation compared with control vector-transfected cells (Figure 4B). In contrast, DU145-ERG cell proliferation in response to incubation with EP3 or EP4 was significantly higher than the control cells at all concentrations tested (Figure 4, B and C), indicating that neither EP3 nor EP4 are involved in the regulation of prostaglandin-mediated proliferation. Taken together with our previous results, only EP2 shows protein overexpression in DU145-ERG cells, as well as a decrease in proliferation after treatment with an EP2 antagonist.

We next cultured DU145 cells transfected with ERG in the presence of prostaglandin-receptor antagonists $(50 \mathrm{nmol} / \mathrm{L}$ each) or the combination of inhibitors and analyzed the effects on IL-6 expression. Culture of ERG-transfected DU145 cells in the presence of all tested prostanoid-receptor antagonists showed a significant reduction in IL-6 expression (Figure 4D). Furthermore, EP2-receptor antagonist alone significantly reduced IL-6 production, underlining the important role of EP2. Culture of DU145-ERG cells in the presence of either EP3 or EP4-receptor antagonist did not modulate IL-6 expression.

To further confirm these findings in three different prostate cancer cell lines we silenced EP2 mRNA expression by transfection with EP2-specific siRNA and analyzed the proliferation and IL-6 production of these cells. All three cell lines 
A

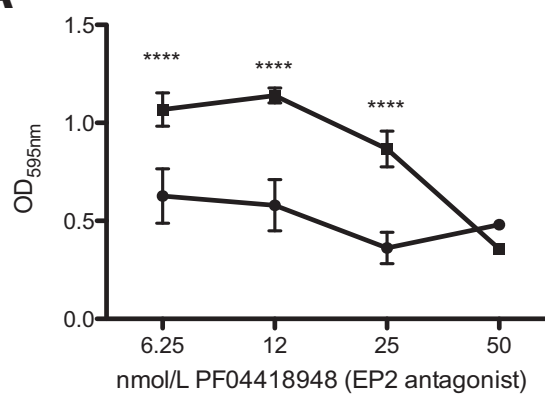

C

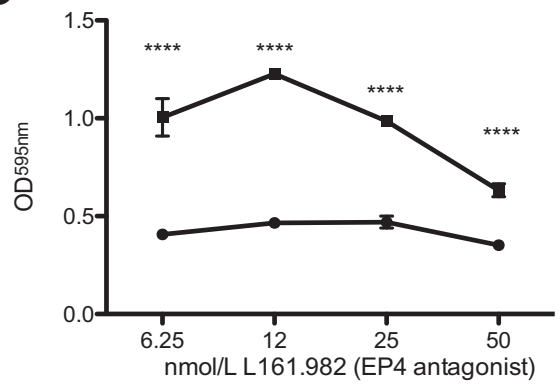

B

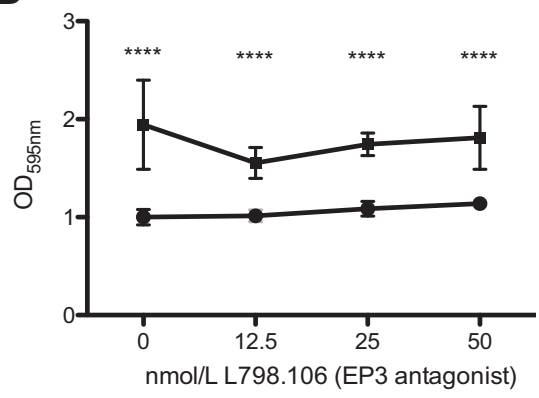

D

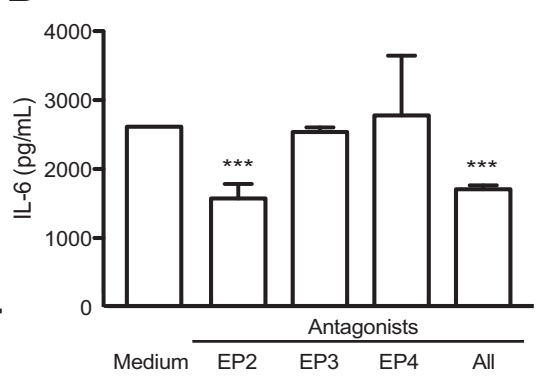

Figure 4 ERG-mediated IL-6 production and proliferation are mediated by the prostanoid receptor EP2. A-C: DU145-ERG (circles) or control vector-transfected DU145 (rectangles) cells were treated for 48 hours with the indicated concentrations of indicated antagonists. Proliferation was measured by MTT assay. D: IL- 6 contents of cell culture supernatants obtained after 48 hours were measured by enzyme-linked immunosorbent assay. E: Indicated cell lines stably transfected with control vector (white bars) or ERG (gray bars) were transiently transfected with EP2-specific scrambled SiRNA and analyzed for their proliferation and IL-6 content, shown as relative values compared with control cells transfected with scrambled siRNA. IL-6 values are shown as floating bars. F: Control vector-transfected DU145 cells were treated for 48 hours with recombinant Pge2. IL-6 contents of cell culture supernatants were measured by enzyme-linked immunosorbent assay and compared with those of ERGtransfected DU145 cells. Results are representative of at least two experiments. $n=3$. ${ }^{*} P<0.01$, ${ }^{* *} P<0.005$, and $* * * * P<0.001$. ERG, erythroblast transformation-specific-related gene.

E

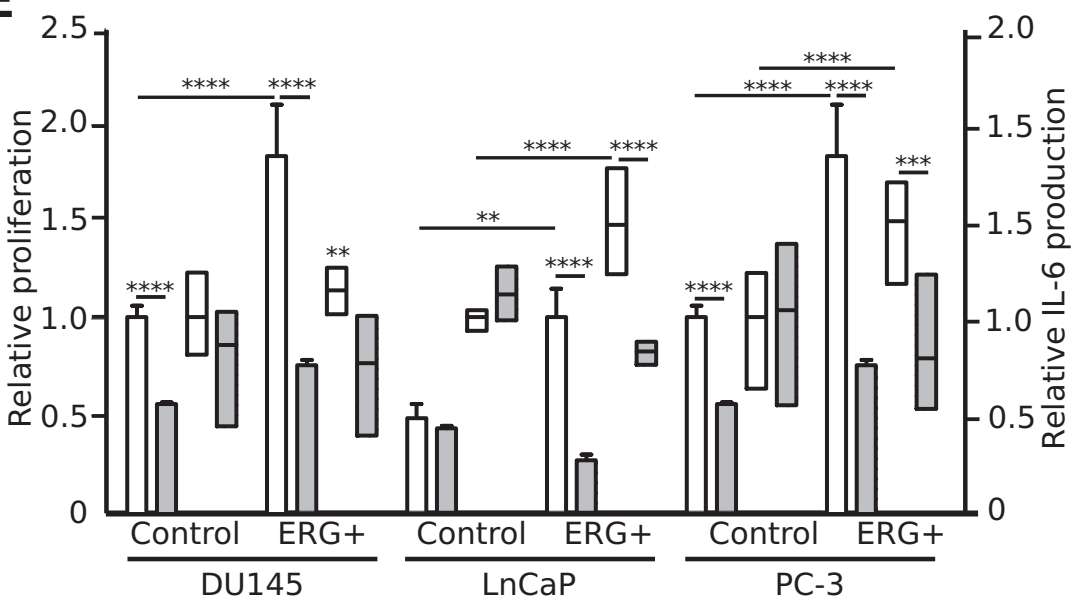

$\mathbf{F}$

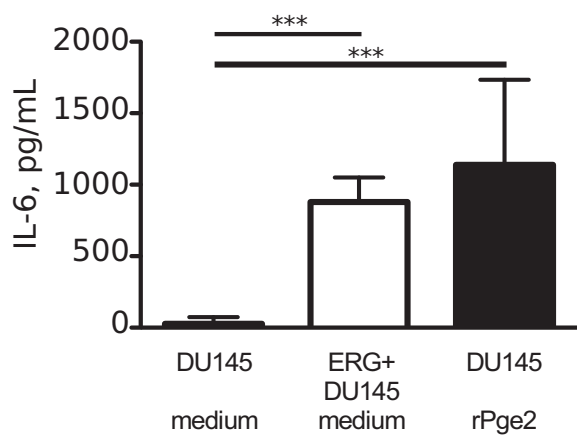

stably transfected with ERG showed increased proliferation as well as IL-6 production compared with control vectortransfected cells (Figure 4E). EP2-knockdown resulted in significantly reduced proliferation and IL-6 production in all ERG-overexpressing cell lines to levels comparable with their respective control cells (Figure 4E). Consequentially, we asked whether prostaglandin as ligand for the prostanoid receptors is sufficient to drive IL-6 expression in prostate tumor cells. To this end, we cultured untransfected DU145 cells in the presence of recombinant Pge 2 or medium alone. The addition of Pge2 was able to increase IL-6 production in DU145 to levels comparable with ERG-transfected DU145 cells (Figure 4F).

\section{ERG Expression Correlates with EP2 Expression}

Given the established effects of prostanoid-receptor inhibition on proliferation and IL-6 expression, we tested the expression of all four receptors in prostate tumor tissue. Tissue microarrays were immunostained against all EP receptors and the frequencies of expressing cells as well as their expression levels were quantified digitally. Neither the frequencies nor the expression levels of the receptors EP1, EP3, or EP4 were significantly different in tumors compared with benign tissues (Figure 5, A, C, and D). In contrast, we observed strong and significant overexpression of EP2 in tumor biopsy specimens compared with benign prostate tissue (Figure 5B). Furthermore, we observed a moderate but significant correlation between the expression level of EP2 in biopsy specimens and the expression of IL-6 (Figure 5F) in the same patient. Moreover, EP2 expression frequency correlated with the frequency of ERGpositive cells (Figure 5F).

\section{Discussion}

In this study we investigated the mechanisms driving expression of IL-6 in PCa tissue and PCa cell lines. Here, 


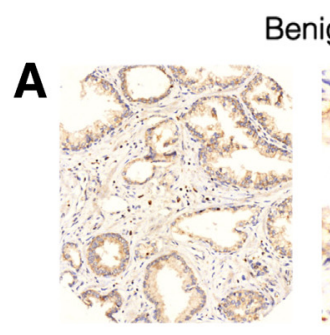

Benign
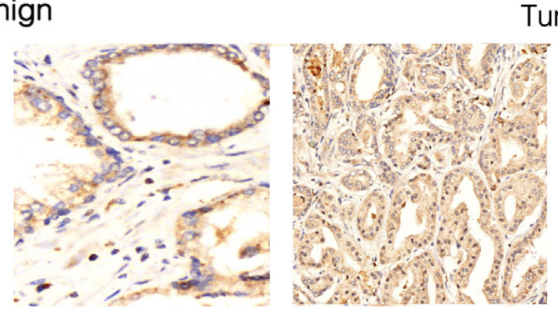

Tumor
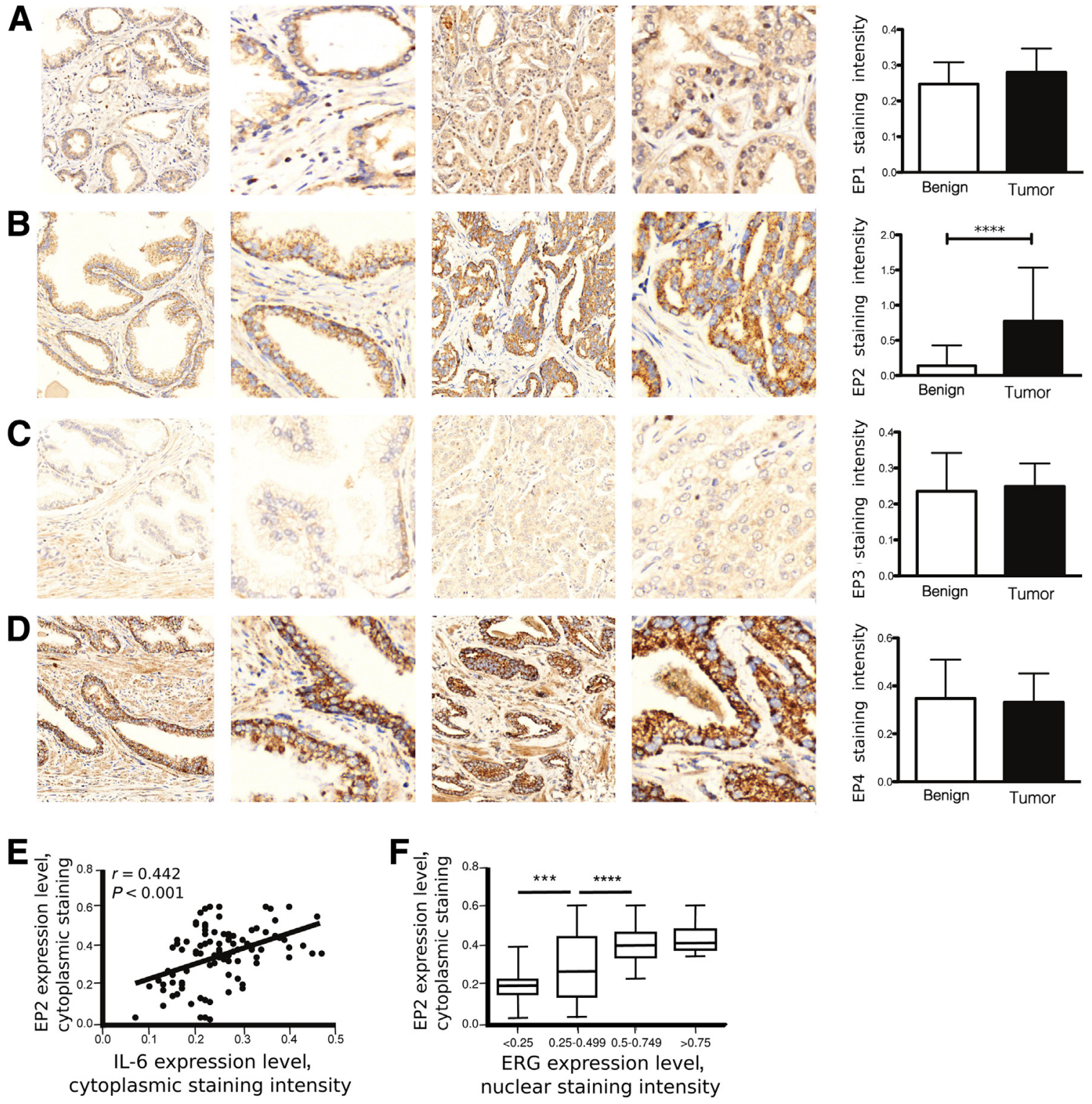

Figure 5 Expression of prostanoid receptor EP2 in prostate tumors correlates with ERG and IL-6. Tissue microarrays containing benign prostate tissue and tumor tissue were stained against EP1 (A), EP2 (B), EP3 (C), or EP4 (D). Protein expression of the respective receptors was quantified and is shown as the frequency of expressing cells of benign prostate tissue or tumor tissue. The cytoplasmic staining intensity of EP2 (mean values per patient) correlated linearly to the mean staining intensities of IL-6 (E) and nuclear expression of ERG (F), of the same patients. $n=34$ (benign); $n=172$ (tumor). ${ }^{* * *} P<0.005,{ }^{* * *} P<0.001$, one-way analysis of variance with the Tukey post-test. ERG, erythroblast transformation-specific-related gene.

we showed that in PCa samples ERG overexpression strongly correlates with IL-6 expression. ERG-positive tissue samples show considerably higher amounts of IL-6 expression than ERG-negative samples.

To explain the strong co-expression of ERG and IL-6 we performed cell culture experiments in PCa cell lines illustrating that ERG expression controls IL-6 secretion: an overexpression in DU145 cells increased IL-6 production, whereas the post-transcriptional inhibition of ERG expression decreased IL-6 production in $\mathrm{VCaP}$ cells, which harbor the $E R G$ translocation. To gain insights into the underlying mechanisms we focused on genes and pathways involved in IL-6 regulation. Testing the expression of a limited number of genes we found HPGD down-regulated in response to ERG overexpression. HPGD is a prostaglandin-catabolizing enzyme, its regulation in prostate cancer remains unclear. Vainio et $\mathrm{al}^{24}$ showed that HPGD mRNA is up-regulated in androgen-independent and metastatic prostate cancer. In contrast, Mohamed et $\mathrm{al}^{20}$ recently reported that ERG directly represses the expression of HPGD in PCa cell lines, which is consistent with the observed down-regulation of HPGD mRNA in response to forced ERG expression. 
In addition, down-regulation of the Pge2-catabolyzing enzyme HPDG resulted in increased levels of Pge2 that were detectable in the supernatants of ERG-overexpressing cells. Moreover, corroborating the high correlation between ERG expression and IL-6 production in prostate tumors, we showed that ERG overexpression is sufficient for the expression of IL-6 and IL- $1 \beta$. Recently, Li et al ${ }^{25}$ showed that IL-1 $\beta$ produced by tumor cells increases the expression of COX-2 in stromal cells, resulting in production of prostaglandin E2. Whether in analogy to this autocrine effect IL-1 $\beta$ up-regulates COX subsequently to Pge 2 or downregulation of HPGD is responsible for increased Pge2 levels remains to be analyzed.

Among the four prostanoid receptors EP1 to EP4, wildtype DU145 cells express only the EP4 subtype. Forced ERG expression results in strongly increased protein expression of EP2 and EP3, although mRNA levels did not change significantly. The apparent discrepancies between mRNA and protein levels of prostanoid receptors have been reported previously. ${ }^{26-28}$ Although comparisons between mRNA and protein levels have to be taken with caution, we speculate that there may be post-transcriptional regulation of the prostanoid receptors. Estrogen is known to regulate the mRNA stability of several genes, including hormone receptors, ${ }^{29}$ and hence appears as a possible regulator.

Both up-regulated receptors EP2 and EP3 appeared as conceivable mediators of the ERG-mediated IL-6 production. A cell growth-supportive function of IL-6 has long been appreciated. ${ }^{30}$ However, the mechanism that drives IL-6 production in cancer is elusive. Consequently, we asked whether blockade of prostanoid receptors in ERG-expressing cells abrogates cell proliferation. We have shown that specific blockade of EP2, a target of ERG overexpression (Figure 3), resulted in abrogation of the proliferative capacity. In contrast, EP3, which also is up-regulated by ERG, seemed not to be involved in cell proliferation. Whether the increased proliferation in response to ERG is mediated by IL-6 resulting from prostanoid-receptor signaling or alternative pathways downstream of EP receptors, remains to be clarified. In silico screening the promoter regions of the four prostanoid receptors for occurrence of ERG-binding sites showed the presence thereof at two positions within a distance of 6.8 upstream of the transcription start of PTGER1 and PTGER2, using a conservative threshold of $90 \%$. In contrast, the ERG binding site motif was not found within the promoter region of either PTGER 3 or PTGER4, which corroborates the up-regulation of PTGER2. Moreover, among the transcripts and proteins tested, IL-1 $\beta$ appeared to be the most stringent regulated target of ERG, and the promotor region of ILIB contains four EBS within a range of $3.4 \mathrm{~kb}$. The number of binding site occurrences does not necessarily reflect differences in regulation of gene expression regulation, however, the significance of these sequence motifs identified in silico remains to be evaluated in vitro.

Previously, in different cellular systems prostanoid receptors were implicated to regulate the cellular processes of tumor cell lines. In these studies, EP3, EP4, or both EP2 and EP4 were required for the prostaglandin-mediated development of castration-resistant prostate cancer, migration, and invasion. ${ }^{20}$ Here, we show that in addition to its effects on proliferation the blockade of either EP1 or EP2 independently resulted in a significant reduction of IL-6 production of ERGexpressing cells. By using primary patient material we could further show that in prostate tumors the receptor subtype EP2 is significantly more highly expressed compared with benign prostate tissue. Collectively, among the four prostanoid receptors, only the EP2 subtype is up-regulated in response to forced ERG expression, both pharmacologic inhibition of EP2 and siRNA-mediated knockdown reduces IL-6 production, and its expression correlates with ERG expression in human prostate tumors. Moreover, analyzing a conceivable association between ERG, IL-6, and EP2, we found moderate correlations among all three proteins in prostate material, thus underlining the mechanistic link between ERG driving the expression of EP2, which induces the subsequent production of IL-6.

\section{References}

1. Birdsey GM, Dryden NJ, Ansellem V, Gebhardt F, Sahnan K, Haskard DO, Dejana E, Mason RC, Randi AM: Transcription factor erg regulates angiogenesis and endothelial apoptosis through VEcadherin. Blood 2008, 111:3498-3506

2. Rahim S, Uren A: Emergence of ETS transcription factors as diagnostic tools and therapeutic targets in prostate cancer. Am J Transl Res 2013, 5:254-268

3. Clinckemalie L, Spans L, Dubois V, Laurent M, Helsen C, Joniau S, Claessens F: Androgen regulation of the TMPRSS2 gene and the effect of a SNP in an androgen response element. Mol Endocrinol 2013, 27: 2028-2040

4. Toubaji A, Albadine R, Meeker AK, Isaacs WB, Lotan T, Haffner MC Chaux A, Epstein JI, Han M, Walsh PC, Partin AQ, De Marzo AM, Platz EA, Netto GJ: Increased gene copy number of ERG on chromosome 21 but not TMPRSS2-ERG fusion predicts outcome in prostatic adenocarcinomas. Mod Pathol 2011, 24:1511-1520

5. Guo Y, Xu F, Lu T, Duan Z: Zhang Z: Interleukin-6 signaling pathway in targeted therapy for cancer. Cancer Treat Rev 2012, 38:904-910

6. Giri D, Ozen M, Ittmann M: Interleukin-6 is an autocrine growth factor in human prostate cancer. Am J Pathol 2001, 159:2159-2165

7. Culig Z, Puhr M: Interleukin-6: a multifunctional targetable cytokine in human prostate cancer. Mol Cell Endocrinol 2012, 360:52-58

8. George DJ, Halabi S, Shepard TF, Sanford B, Vogelzang NJ, Small EJ Kantoff PW: The prognostic significance of plasma interleukin-6 levels in patients with metastatic hormone-refractory prostate cancer: results from cancer and leukemia group B 9480. Clin Cancer Res 2005, 11: $1815-1820$

9. Palmer J, Hertzog PJ, Hammacher A: Differential expression and effects of gp130 cytokines and receptors in prostate cancer cells. Int J Biochem Cell Biol 2004, 36:2258-2269

10. Grubbs CJ, Lubet RA, Koki AT, Leahy KM, Masferrer JL, Steele VE, Kelloff GJ, Hill DL, Seibert K: Celecoxib inhibits N-butyl-N-(4hydroxybutyl)-nitrosamine-induced urinary bladder cancers in male B6D2F1 mice and female Fischer-344 rats. Cancer Res 2000, 60: $5599-5602$

11. Reader J, Holt D, Fulton A: Prostaglandin E2 EP receptors as therapeutic targets in breast cancer. Cancer Metastasis Rev 2011, 30:449-463

12. Ma J, Chen M, Xia SK, Shu W, Guo Y, Wang YH, Xu Y, Bai XM, Zhang L, Zhang L, Zhang M, Wang YP, Leng J: Prostaglandin E2 
promotes liver cancer cell growth by the upregulation of FUSE-binding protein 1 expression. Int J Oncol 2013, 42:1093-1104

13. Wu T: Cyclooxygenase- 2 and prostaglandin signaling in cholangiocarcinoma. Biochim Biophys Acta 2005, 1755:135-150

14. Wu J, Zhang Y, Frilot N, Kim JI, Kim WJ, Daaka Y: Prostaglandin e 2 regulates renal cell carcinoma invasion through the EP4 receptor-rap GTpase signal transduction pathway. J Biol Chem 2011, 286: 33954-33962

15. Vo B, Morton D Jr, Komaragiri S, Millena AC, Leath C, Khan SA: TGF- $\beta$ effects on prostate cancer cell migration and invasion are mediated by PGE2 through activation of PI3K/AKT/mTOR pathway. Endocrinology 2013, 154:1768-1779

16. Kashiwagi E, Shiota M, Yokomizo A, Itsumi M, Inokuchi J, Uchiumi T, Naito S: Prostaglandin receptor EP3 mediates growth inhibitory effect of aspirin through androgen receptor and contributes to castration resistance in prostate cancer cells. Endocr Relat Cancer 2013, 20:431-441

17. Huang HF, Shu P, Murphy TF, Aisner S, Fitzhugh VA, Jordan ML: Significance of divergent expression of prostaglandin EP4 and EP3 receptors in human prostate cancer. Mol Cancer Res 2013, 11: 427-440

18. Nowak M, Svensson MA, Carlsson J, Vogel W, Kebschull M, Wernert N, Kristiansen G, Andrén O, Braun M, Perner S: Prognostic significance of phospho-histone $\mathrm{H} 3$ in prostate carcinoma. World $\mathrm{J}$ Urol 2014, 32:703-707

19. Sandelin A: JASPAR: an open-access database for eukaryotic transcription factor binding profiles. Nucleic Acids Res 2004, 32: 91D-94D

20. Mohamed AA, SH Tan, Sun C, Shaheduzzaman S, Hu Y, Petrovics G, Chen Y, Sesterhenn IA, Li H, Sreenath T, McLeod DG, Dobi A, Srivastava S: ERG oncogene modulates prostaglandin signaling in prostate cancer cells. Cancer Biol Ther 2011, 11:410-417

21. Allan EH, Martin TJ: Prostaglandin E2 regulates production of plasminogen activator isoenzymes, urokinase receptor, and plasminogen activator inhibitor-1 in primary cultures of rat calvarial osteoblasts. J Cell Physiol 1995, 165:521-529

22. Dinarello CA: Interleukin-1 and the effects of cyclooxygenase inhibitors on its biological activities. Bull N Y Acad Med 1989, 65:80-92

23. Karim FD, Urness LD, Thummel CS, Klemsz MJ, McKercher SR, Celada A, Van Beveren C, Maki RA, Gunther CV, Nye JA, Graves BJ: The ETS-domain: a new DNA-binding motif that recognizes a purinerich core DNA sequence. Genes Dev 1990, 4:1451-1453

24. Vainio P, Gupta S, Ketola K, Mirtti T, Mpindi JP, Kohonen P, Fey V, Perälä M, Smit F, Verhaegh G, Schalken J, Alanen KA, Kallioniemi O, Iljin K: Arachidonic acid pathway members PLA2G7, HPGD, EPHX2, and CYP4F8 identified as putative novel therapeutic targets in prostate cancer. Am J Pathol 2011, 178: $525-536$

25. Li HJ, Reinhardt F, Herschman HR, Weinberg RA: Cancer-stimulated mesenchymal stem cells create a carcinoma stem cell niche via prostaglandin E2 signaling. Cancer Discov 2012, 2:840-855

26. Hinton AC, Grigsby PL, Pitzer BA, Brockman DE, Ittenbach RF, Hinton RB, Myatt L: Hormonal regulation of prostaglandin E2 receptors: localization and expression in rat cervical tissue. Reprod Sci 2010, 17:136-146

27. Catalano RD, Wilson MR, Boddy SC, Jabbour HN: Comprehensive expression analysis of prostanoid enzymes and receptors in the human endometrium across the menstrual cycle. Mol Hum Reprod 2011, 17: $182-192$

28. Blesson CS, Büttner E, Masironi B, Sahlin L: Prostaglandin receptors $\mathrm{EP}$ and FP are regulated by estradiol and progesterone in the uterus of ovariectomized rats. Reprod Biol Endocrinol 2012, 10:3

29. Ing NH: Steroid hormones regulate gene expression posttranscriptionally by altering the stabilities of messenger RNAs. Biol Reprod 2005, 72:1290-1296

30. Klein B, Zhang XG, Jourdan M, Boiron JM, Portier M, Lu ZY, Wijdenes J, Brochier J, Bataille R: Interleukin-6 is the central tumor growth factor in vitro and in vivo in multiple myeloma. Eur Cytokine Netw 1990, 1:193-201 